\title{
A CONTACTLESS ELECTROCHEMICAL IMPEDANCE MEASUREMENT METHOD
}

\author{
L.Yu, E. Pun, and E. Meng \\ University of Southern California, Los Angeles, California, USA
}

\begin{abstract}
We present the first demonstration of a passive, contactless method to interrogate electrochemical impedance (EI) via a microfabricated MEMS coil. EI measurement was achieved using the principle of reflected impedance between a pair of inductively coupled coils. Ionic concentration and electrolyte temperature were wirelessly transduced from measurement electrodes connected to a microfabricated coil in a flexible thin film substrate. This technique is widely applicable for resistive transduction and obviates the need for soldering discrete electronic components, microcontrollers, batteries, and bulky hermetic packaging. It also features low power consumption $(\sim 75 \mu \mathrm{W})$ and all biocompatible construction (only $\mathrm{Au}, \mathrm{Ti}$, and Parylene $\mathrm{C}$ ), making it ideal for use in harsh or in vivo environments.
\end{abstract}

\section{INTRODUCTION}

Chronic recording of physiological signals via the use of implanted sensors has been a long-standing goal for the medical and scientific community. Such sensors would enable continuous monitoring to facilitate greater understanding of disease progression as well as inform course of treatment.

The in vivo environment poses a formidable challenge for the development of telemetry circuitry used to encode and wirelessly transmit measured signals [1]. Water vapor and ion permeation create undesired paths for electric current and can alter critical bias voltages. The inflammatory response to foreign bodies can subject the implant to a highly oxidative or acidic environment [2]. Blood flow and other involuntary movements can subject the implant to chronic and repeated mechanical strain. Such events can damage sensitive components or introduce errors in signal measurement and transmission.

Naturally, the presence of the implant should also minimize harm to the host environment. The materials selected for the packaged device should be nontoxic and induce minimal immune response. The use of soft, flexible materials (i.e. those mechanically matching tissue properties) and elimination of sharp corners also serves to minimize damage to tissue [3]. Power requirements for electronics should be kept in check to minimize heating to safe levels.

In this work, our goal is to address these requirements with a new method to measure and transmit signals within a simulated in vivo environment. Passive wireless capacitive transduction methods have been widely studied and utilized, with notable examples demonstrating successful chronic implantation in humans [4]. However, for resistive transduction, wireless transmission has been limited to the use of active circuitry (analog or digital via microcontrollers). This burdens system design by adding power constraints, thermal budget concerns, and bulk to the overall package. Here, we introduce the use of reflected impedance across a pair of inductively coupled coils to achieve wireless resistive transduction without active circuitry and demonstrated its use for measurement of electrolyte conductivity and temperature.

\section{THEORETICAL CONSIDERATIONS}

A pair of inductively coupled coils shares the properties of a transformer. An alternating current source induces a varying magnetic field in the primary coil, which then induces a varying electromotive force in the secondary coil (Figure 1).

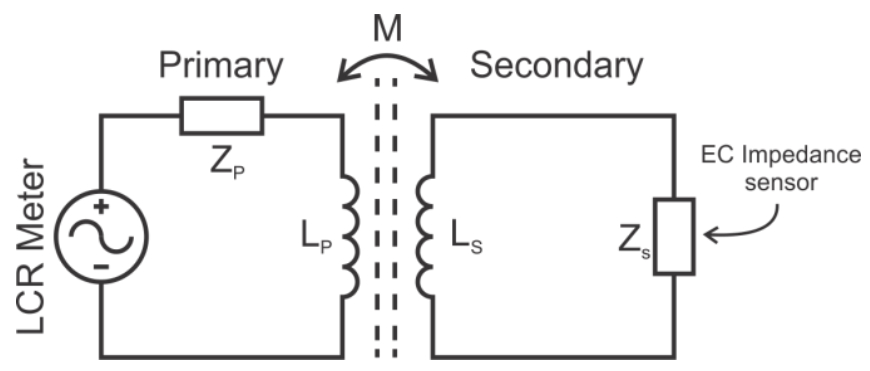

Figure 1: Schematic of reflected impedance principle, where $Z_{p}$ represents the complex impedance of the primary circuit resistance and capacitance, $L_{p}$ and $L_{s}$ the inductances of coupled coils, and $Z_{s}$ the complex impedance of the load on the secondary circuit. An AC source (LCR meter) induces current within the secondary circuit across a pair of inductively coupled coils (coupling coefficient $M$ ).

To simplify, an equivalent single loop model that lumps the secondary circuit into a complex impedance term may be utilized (Figure 2). Due to conservation of energy, a load that is attached to the secondary coil will result in an apparent load on the primary side, as represented in (1). In this manner, changes in the secondary load may be reflected across the inductively coupled coils and measured as changes on the primary side [5].

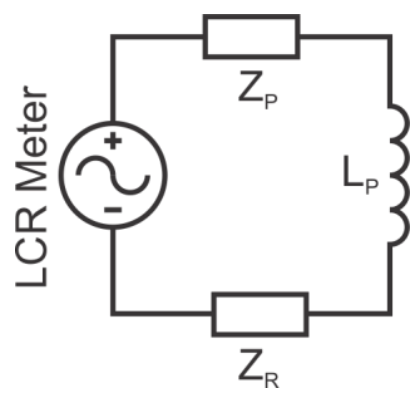

Figure 2: Equivalent single loop primary circuit with reflected impedance represented as $Z_{R}$.

$$
Z_{R}=\frac{\omega^{2} M^{2}}{Z_{s}+j \omega L_{s}}
$$

\section{DESIGN}

Size of the secondary coil was maximized to optimize coupling with the primary coil. Coupling can also be improved by increasing the number of turns in order to capture increased magnetic flux. However, this can result in a large and unwieldy device footprint for implant applications. Additional improvement in coupling can be achieved through the use of a ferrite core on the primary coil.

All of the materials selected for use have a proven track record for use within the in vivo environment. Thin film gold was used for the electrodes due to its inertness and its well-characterized electrochemical properties [6]. Electrode size and spacing were 
based on previous investigations on electrochemical impedance sensing [7]. The low resistivity of gold also serves advantageous for its use as a coil in RF applications. Parylene $\mathrm{C}$ was selected as the substrate and to encapsulate the electrodes and coil, due to its excellent insulation properties as well as its amenability to microfabrication processes [8].

\section{METHODS}

The fabrication of this thin film polymer device was based on previously reported methods [7]. A pair of Au electrodes (2000 $\AA$ thick on $200 \AA$ Ti adhesion layer, $300 \mu \mathrm{m} \times 300 \mu \mathrm{m}, 500 \mu \mathrm{m}-5000$ $\mu \mathrm{m}$ electrode-to-electrode spacing) attached to a planar coil $(30 \mathrm{~mm}$ diameter, 1-16 turns) was patterned and electron beam evaporated onto a flexible Parylene $\mathrm{C}$ substrate (10 $\mu \mathrm{m}$ thick) (Figure 3). Following deposition of another $10 \mu \mathrm{m}$ Parylene insulation layer, electrodes were exposed via $\mathrm{O}_{2}$ plasma reactive ion etching (Technics RIE, $200 \mathrm{~W}, 150$ mTorr) [9]. (i)

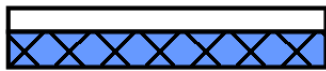

(iii)

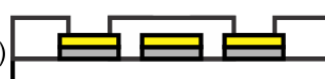

(ii)

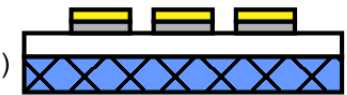

Figure 3: Overview of fabrication process. (i) Deposition of $10 \mu \mathrm{m}$ thick Parylene $C$ substrate. (ii) Patterning and deposition of Ti adhesion layer and Au. (iii) Deposition of $10 \mu \mathrm{m}$ Parylene insulation layer, reactive ion etching $\left(\mathrm{O}_{2}\right)$ to expose electrode sites, release, and cutout from silicon substrate.

After cutout and release (Figure 4), the devices were placed in a polystyrene Petri dish and submerged in phosphate buffered saline (PBS) of various concentrations $(0.5-10 \times, 8-150 \mathrm{mS} / \mathrm{cm} \mathrm{[10])} \mathrm{and}$ temperatures $\left(22^{\circ} \mathrm{C}-65^{\circ} \mathrm{C}\right)$ to mimic a range of typical and abnormal in vivo conditions. Deionized water was used to establish a baseline impedance reference.

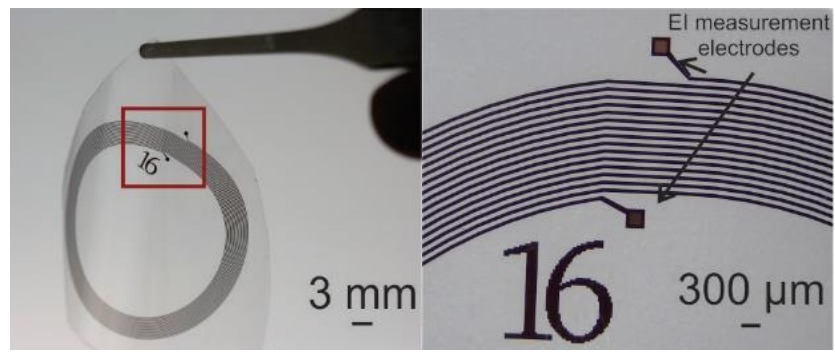

Figure 4: (Left) Photograph of microfabricated and released thin film Parylene $C$ device held by forceps. Sixteen turn coil shown; one, five, and six turn coils were also fabricated. (Right) Inset of left; micrograph highlighting electrochemical impedance (EI) measurement electrodes $(300 \times 300 \mu$ m exposed area $)$.

The secondary circuit comprises of the Au planar coil $(L=11.1$ $\mu \mathrm{H}, Q \sim 1.5$ at $2.198 \mathrm{MHz}$ ) and electrodes submerged in electrolyte, which can be represented by the Randles circuit model (Figure 5). At resonant frequency, the reactance of the capacitive $\left(C_{d l}\right)$ and inductive (thin film coil) elements will be equal in magnitude but opposite in sign. The resulting reflected impedance consists of the remaining resistive element, which is the solution resistance $\left(R_{S}\right)$. The Petri dish $(0.8 \mathrm{~mm}$ thick base $)$ was placed onto and concentrically aligned with the commercially available primary coil having a ferrite core (Figure 6, $24 \mu \mathrm{H}, Q=180 @ 125 \mathrm{kHz}$, Wurth Electronics, $50 \mathrm{~mm}$ diameter, 22 turns).

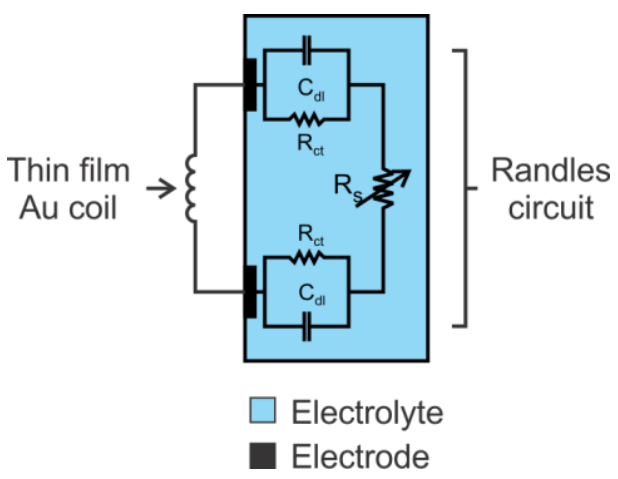

Figure 5: Secondary circuit schematic, which comprises of insulated Au coil and Randles circuit model of electrodes submersed in electrolyte.

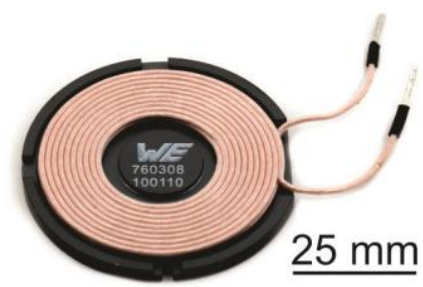

Figure 6: Primary coil with ferrite core to improve coupling with thin film (secondary) coil.

The primary coil was elevated (onto an additional Petri dish to prevent inadvertent coupling with the benchtop) and attached to an LCR meter (Hewlett Packard E4285A) (Figure 7), which provided the AC signal and recorded the reflected impedance.

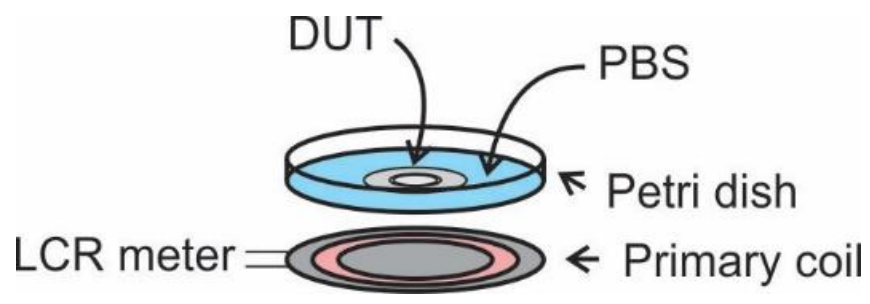

Figure 7: Device under test (DUT) was placed within a Petri dish and submerged in PBS (phosphate buffered saline). Primary coil includes ferrite core to improve inductive coupling with thin film (secondary) coil. Not drawn to scale.

The optimal measurement frequency was determined to be $2.198 \mathrm{MHz}\left(Q=34.3\right.$, minimum phase, $\left.1 \mathrm{~V}_{\mathrm{p}-\mathrm{p}}\right)$ to bypass the double layer capacitance $\left(C_{d l}\right)$ in the Randles circuit (Figure 8) [11]. At this measurement frequency, the impedance is dominated by the solution resistance. Reproducibility of coil position was achieved by observing phase angle measurement and adjusting alignment until minimum phase $\left(< \pm 1^{\circ}\right)$ was attained. To assess misalignment performance, coil centers were translated up to $5 \mathrm{~mm}$ from initial alignment while measuring reflected impedance. 


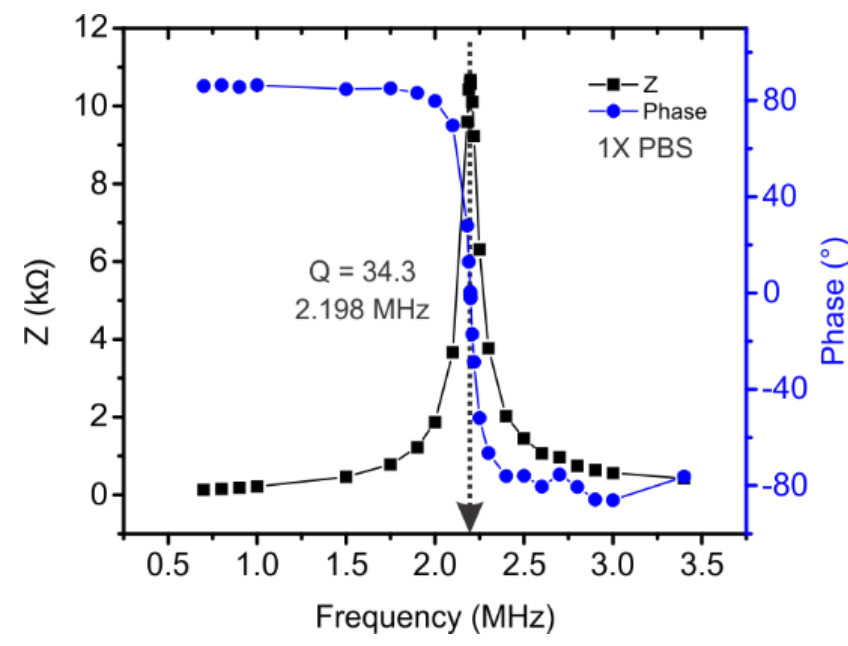

Figure 8: Frequency response of system comprising of the primary coil coupled with the DUT. The excitation signal on the LCR meter was set to $2.198 \mathrm{MHz}$ (minimum system phase to bypass double layer capacitance $C_{d l}$ ) for subsequent testing.

\section{RESULTS}

Reflected electrochemical impedance was measured from fabricated devices and utilized to transduce ionic concentration and temperature of the electrolyte into which the devices were submerged (Figure 9). The observed power draw at the primary coil was approximately $75 \mu \mathrm{W}$.

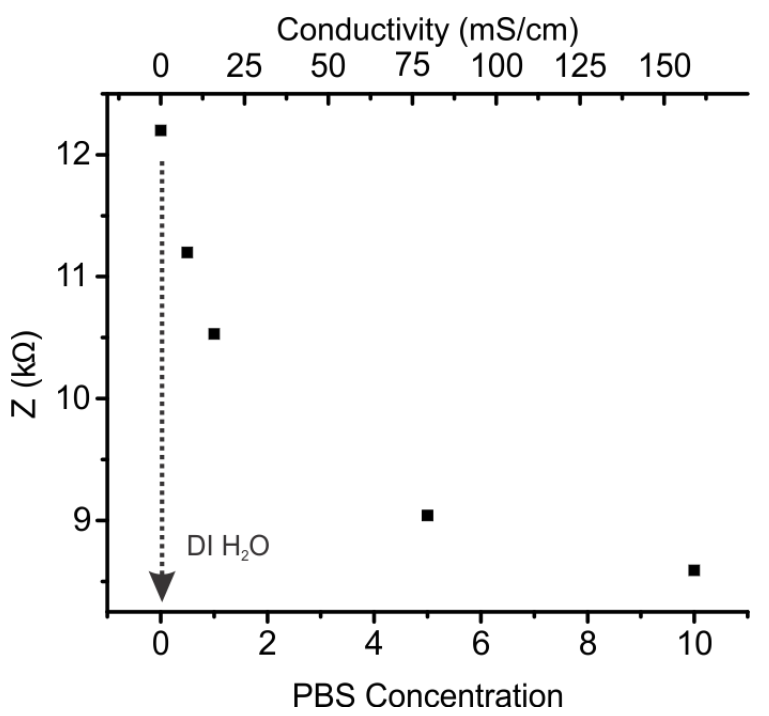

Figure 9: Reflected impedance was observed to be inversely proportional to ionic concentration. Deionized water was used as a baseline reference and indicated using the arrow.

Electrochemical impedance of ionic solutions has previously been shown with a direct wired measurement to be inversely proportional to temperature [12]. This relation was measured wirelessly using this reflected impedance method with $1 \times$ PBS across a range of different temperatures (Figure 10).

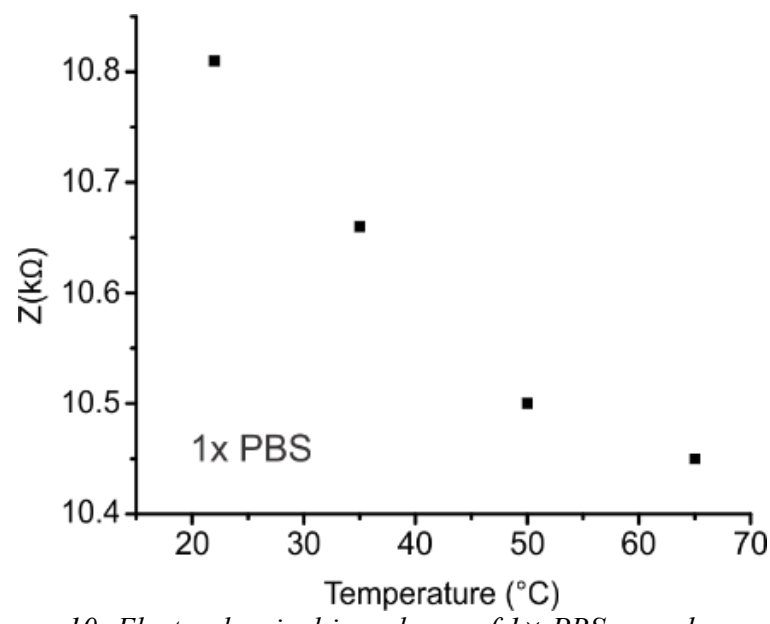

Figure 10: Electrochemical impedance of $1 \times P B S$ was observed to be inversely proportional to temperature.

Reflected impedance increased linearly with coil misalignment by approximately $1 \%$ per mm of coil center offset. The results were achieved with a 5 turn thin film coil, and preliminary results suggest that altering coil diameter and increasing turn count can reduce misalignment effects on baseline impedance measurement.

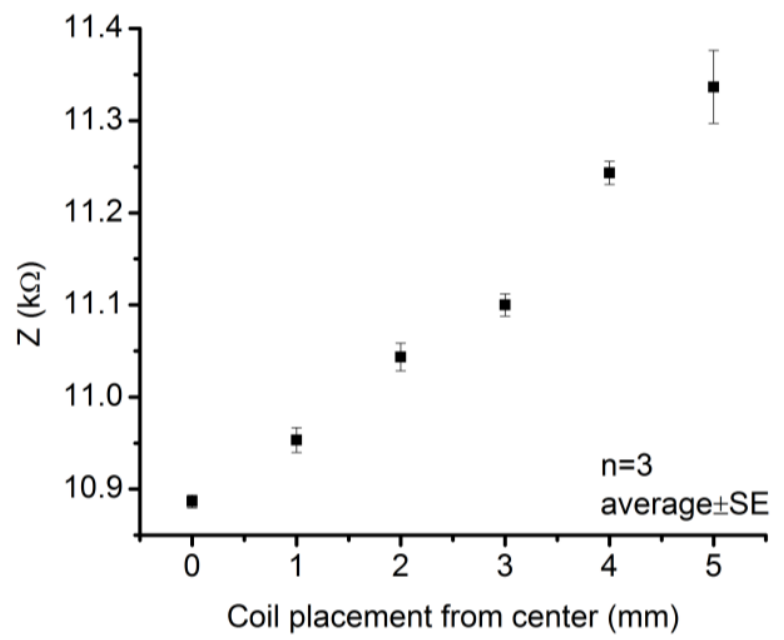

Figure 11: Reflected impedance increased with coil misalignment across the range tested, but remained within 5\% of baseline measurement.

\section{CONCLUSION}

A method to wirelessly measure electrochemical impedance was developed and demonstrated by measuring ionic conductivity $(0-150 \mathrm{mS} / \mathrm{cm})$ and solution temperature $\left(22-65^{\circ} \mathrm{C}\right)$. The method utilizes inductively coupled coils and requires no active electronic components. Measurement frequency was determined to be 2.198 $\mathrm{MHz}$ and performance with suboptimal coil alignment (translation up to $5 \mathrm{~mm}$ ) was evaluated. Featuring all biocompatible material construction, flexible polymer substrate, and low power draw $(<100 \mu \mathrm{W})$, the device is well suited for the wet, in vivo environment. The overall structure may also lend itself well to use in other harsh environments.

The electrochemical impedance transduction principle utilized here was previously demonstrated in a variety of sensing applications such as sensing of catheter blockage [13], liquid flow measurement [12], contact force [7], and drug delivery volume [10, 14], but with direct wired measurements. We demonstrated that it 
may be feasible to incorporate a coil and adjust electrode packaging to enable wireless subcutaneous sensing of these aforementioned signals. In addition, coating the electrode surface with antigens [15] or chemically sensitive polymers [16] can add specificity to the electrochemical measurement.

Additional development is planned to refine this method for implant applications. Miniaturization of coil size and further characterization of inductive coupling (foveation, distance, increasing $\mathrm{Q}$ factor) will be performed. Performance will be assessed in an ex vivo application to measure catheter obstruction.

\section{ACKNOWLEDGEMENTS}

This work was funded by the NSF under award number ECCS1231994. Travel support was provided by OAI. An OAI hybralign series 200 mask aligner was used for the processing of Parylene devices. The authors would like to thank Dr. Kee Scholten, Dr. Donghai Zhu, and the members of the USC Biomedical Microsystems Laboratory for their assistance.

\section{REFERENCES}

[1] L. Bowman and J. D. Meindl, "The packaging of implantable integrated sensors," Biomedical Engineering, IEEE Transactions on, pp. 248-255, 1986.

[2] J. M. Anderson, A. Rodriguez, and D. T. Chang, "Foreign body reaction to biomaterials," in Seminars in immunology, 2008, pp. 86-100.

[3] F. Silver, Y. Kato, M. Ohno, and A. Wasserman, "Analysis of mammalian connective tissue: relationship between hierarchical structures and mechanical properties," Journal of long-term effects of medical implants, vol. 2, pp. 165-198, 1991.

[4] M. Fonseca, M. Allen, D. Stern, J. White, and J. Kroh, "Implantable wireless sensor for pressure measurement within the heart," U.S. Patent 6855115, February 15, 2005.

[5] J. R. Long, "Monolithic transformers for silicon RF IC design," Solid-State Circuits, IEEE Journal of, vol. 35, pp. 1368-1382, 2000.

[6] G. Li and P. Miao, "Theoretical background of electrochemical analysis," in Electrochemical Analysis of Proteins and Cells, ed: Springer, 2013, pp. 5-18.

[7] C. A. Gutierrez, C. McCarty, B. Kim, M. Pahwa, and E. Meng, "An Implantable All-Parylene Liquid-Impedance Based MEMS Force Sensor," in MEMS 2010: 23rd IEEE International Conference on Micro Electro Mechanical Systems, Technical Digest, ed New York: IEEE, 2010, pp. 600-603.

[8] E. Meng and Y.-C. Tai, "Parylene etching techniques for microfluidics and bioMEMS," in Micro Electro Mechanical Systems, 2005. MEMS 2005. 18th IEEE International Conference on, 2005, pp. 568-571.

[9] E. Meng, P.-Y. Li, and Y.-C. Tai, "Plasma removal of Parylene C," Journal of Micromechanics and Microengineering, vol. 18, p. 045004, 2008.

[10] A. M. Johnson, D. R. Sadoway, M. J. Cima, and R. Langer, "Design and testing of an impedance-based sensor for monitoring drug delivery," Journal of the Electrochemical Society, vol. 152, pp. H6-H1 1, 2005.

[11] J. E. B. Randles, "Kinetics of Rapid Electrode Reactions," Discussions of the Faraday Society, vol. 1, pp. 11-19, 1947.

[12] A. Baldwin, L. Yu, and E. Meng, "An Electrochemical-Based Thermal Flow Sensor," presented at the 29th IEEE International Conference on Micro Electro Mechanical
Systems, MEMS 2016, January 24 - January 28, 2016 Shanghai, China, 2016.

[13] B. J. Kim, W. Jin, L. Yu, and E. Meng, "MEMS electrochemical patency sensor for detection of hydrocephalus shunt obstruction," in Micro Electro Mechanical Systems (MEMS), 2015 28th IEEE International Conference on, 2015, pp. 662-665.

[14] C. A. Gutierrez, R. Sheybani, and E. Meng, "Electrochemically-based dose measurement for closed-loop drug delivery applications," in Solid-State Sensors, Actuators and Microsystems Conference (TRANSDUCERS), 2011 16th International, 2011, pp. 2839-2842.

[15] S. Song, R. A. Clark, E. F. Bowden, and M. J. Tarlov, "Characterization of cytochrome c/alkanethiolate structures prepared by self-assembly on gold," The Journal of Physical Chemistry, vol. 97, pp. 6564-6572, 1993.

[16] W. O. Ho, S. Krause, C. J. McNeil, J. A. Pritchard, R. D. Armstrong, D. Athey, et al., "Electrochemical sensor for measurement of urea and creatinine in serum based on ac impedance measurement of enzyme-catalyzed polymer transformation," Analytical Chemistry, vol. 71, pp. 19401946, 1999.

\section{CONTACT}

E. Meng, tel: +1-213-740-6952; ellis.meng@usc.edu 